\title{
Compte rendu des travaux océanographiques exposés à l'Assemblée générale de l'A.I.O.P. à Toronto
}

\author{
Report on oceanographic papers read \\ at the Toronto general Assembly of the I.A.P.O.
}

\author{
PAR H. LACOMBE
}

PROFESSEUR D'OCÉANOGHAPHE PHYSTQUE AU MUSÉUM D'HISTOIRE NATURELLE, INGKNEUR HYDROGRAPHE EN CHEF DE LA MARINE (H.C.)

\begin{abstract}
'L'auteur présente' ici un rapide exposé des principaux points abordés dans des communications faites devant l'assemblée générale de l'Association Internationale d'Océanographie Physique tenue à Toronto (Canada) du 3 au 14 septembre 1957. Le premier symposium, sur le « bilan d'eau », a été l'objet d'une réunion conjointe avec l'Association Internationale de Météorologie et a illustré, comme aussi le symposium sur les échanges de quantités de mouvement entre l'atmosphère et l'océan, le besoin essentiel d'une liaison entre océanographes et météorologistes, puisque les phénomènes marins sont. pour une très grande part, conditionnés par l'énergie franchissant l'interface air-eal. Outre cet important aspect, les symposiums étaient centrés sur les autres grandes préoccupations de l'océanographie physique moderne, turbulence près du fond de la mer, circulation océanique profonde, marées au large.

En outre, des études sur des sujets océanographiques divers ont été présentées.
\end{abstract}

\begin{abstract}
The author briefly describes the main points which arose in the papers presented at the General Assembly of the International Association of Physical Oceanography, held at Toronto from September 3rd to 14th 1957. The first symposium, on the "water balance sheet," was the occasion for a joint meeting with the World Meteorological Association and, like the symposium on the exchange of momentum between the atmosphere and the ocean, showed the great need for liaison between oceanographers and meteorologists since marine phenomena are largely conditioned by the energ!l which passes betwen the air and the water. In addition to this important question, the slymposiums dealt with other important problems of modern physical oceanography, furbulence near the sea bed, oceanic circulation at great depths and tides in the open sea.

Papers on various other oceanographical topics were also presented.
\end{abstract}

La $\mathrm{XI}^{\mathrm{e}}$ Assemblée générale de l'Association Internationale d'Océanographie Physique, au sein de l'Assemblée générale de l'Union Géodésique et Géophysique Internationale, s'est tenue à Toronto (Canada) du 3 au 14 septembre 1957 . L'assemblée s'est tenue à l'Université de Toronto.

De nombreuses réunions scientifiques se sont déroulées à cette occasion. Elles ont porté :

D'une part, sur des symposiums :

a) Le bilan d'eau (Water Balance) au cours d'une réunion conjointe avec l'Association Internationale de Météorologie; b) La circulation océanique et particulièrement la circulation abyssale;

c) Le transfert de quantité de mouvement de l'atmosphère à l'océan;

d) La construction des cartes cotidales dans les océans;

e) Les courants de marée près du fond, dans les eaux côtières et les estuaires.

D'autre part, sur des sujets océanographiques divers. 


\section{a) Symposium sur le bilan d'eau :}

a) L'étude du bilan d'eau fut l'occasion pour le Professeur $H$. Mosby de traiter, dans son adresse présidentielle, des divers modes d'attaque du difficile problème de l'évaporation des océans, si importante pour les météorologistes et les océanographes.

Les recherches poursuivies n'ont pas encore abouti à des résultats bien sûrs; en utilisant le bilan d'eau total du slobe, on peut avoir des ordres de grandeur d'ensemble pour les océans, sous réserve de mesures d'évaporation à terre (plus facile qu'à la mer), de débit des fleuves et de précipitations à terre et à la mer (celles-ci plus difficiles); mais on a peu d'informations sur le régime des régions localisées.

Les mesures directes d'évaporation dans des bacs portés par les navires sont sujettes à d'importantes erreurs, amenant à des valeurs trop élevées de l'évaporation, en raison du fait que la vitesse du vent à la surface même de l'eau est inférieure à celle qué l'on mesure à quelques mètres au-dessus de ce niveau et parce que le degré hygrométrique varie avec l'altitude. Mais l'étude du transfert d'eau et du transfert de chaleur à travers la surface de l'eau, dans lesquels interviennenit la diffusion turbulente de la vapeur d'eau et de la chaleur, le degré hygrométrique et thermique, la vitesse du vent dans les couches voisines de la surface, etc., permettent d'attaquer ce problème par des voies théoriques, comportant diverses approximations, mais qui ont fourni quelques ordres de grandeur et ont été employées pour corriger les valeurs mesurées dans des bacs portés par les navires. Wust a trouvé ainsi que, en fonction de la latitude, la salinité superficielle des mers avait une variation en liaison étroite avec la différence « précipitation moins évaporation » et il en a déduit un tableau des valeurs de l'évaporation en fonction de la latitude. Mais pour améliorer nos connaissances, des mesures plus précises devraient être conduites qui porteraient sur les précipitations, les températures (de l'air et de l'eau de surface) et le degré hygrométrique (psychromètre à aspiration), mesures qui sont presque toutes du domaine du météorologiste, et dont je ne ferai que signaler l'extrême intérêt pour l'océanographe. Le $D^{r}$ Wust, l'éminent océanographe allemand, estime que l'organisation de telles mesures est très souhaitable et aussi très possible.

Le $D^{r}$ Sutcliffe, essentiellement météorologiste, se place au-dessus du sol en vue d'une étude de la circulation de l'humidité dans l'atmosphère qui est nécessaire pour égaliser les différences entre l'évaporation et les précipitations, selon les régions.

Les chercheurs australiens (Swinbank) ont apporté une substantielle contribution à la me- sure de l'évaporation des océans, au moyen du bilan d'énergie, à supposer connu le rapport, dit de Bowen, de l'énergie théorique perdue par évaporation à l'énergie perdue par conduction thermique (valeur de 0,1 à 0,2 ). Mais, en raison de la difficulté d'apprécier l'énergie reçe par la mer, les auteurs pensent que la méthode en question comporte une part d'incertitude et préfèrent la suivante.

L'estimation de l'évaporation peut aussi être faite par l'hydrodynamique, c'est-à-dire par l'étude du transfert d'eau au voisinage de la surface de l'eau par diffusion turbulente de la vapeur d'eau. Si on admet que le coefficient de diffusion turbulente est égal au coefficient de transfert de quantité de mouvement [coefficient de viscosité virtuelle vertical (1)] que l'on peut, lui, obtenir à partir d'un profil du vent près de la surface marine, on peut déduire l'évaporation de mesures de vent et de teneur de l'air en vapeur d'eau $[\mathrm{E}=-\mathrm{A}(d q / d t) ; \tau=-\mathrm{A}(d v / d t)]$ ( $q$ teneur en eau, $v$ vitesse du vent).

Des difficultés subsistent concernant le coefficient de viscosité virtuelle, mais par l'étude des corrélations entre les fluctuations de $q$ et des vitesses verticales de vent, les auteurs pensent avoir obtenu ou, en tout cas, pouvoir obtenir des valeurs st̂res.

A.C. Redfield présenta ensuite une étude (faite avec I. Friedman) sur la distribution du deutérium dans les Océans Arctique et Allantique, qui se rapproche du sujet du «bilan d'eau » en raison de la variation du contenu en deutérium selon l'origine de l'eau qui le contient : ainsi la tension de vapeur de l'eau lourde est inférieure de $7 \%$ à celle de l'eau et la vapeur d'eau est moins riche en eau lourde que l'eau dont elle provient, qui s'enrichit donc en deutérium. Aussi paraît-il possible de distinguer l'eau diluée par des précipitations, de l'eau diluée par la fonte des glaces, par sa teneur en deutérium ou plutôt par la corrélation entre la teneur en deutérium et la salinité. Le deutérium constitue donc un traceur pour suivre le mouvement de l'eau dans son cycle entre l'atmosphère, les eaux terrestres et la mer.

Si dans les eaux océaniques profondes de l'Arctique et de l'Atlantique (et sans doute aussi de l'Antarctique) la teneur en deutérium varie fort peu, il n'en est pas de même pour les eaux moins profondes dont la teneur varie notablement et semble caractéristique du bassin.

Le symposium s'achève sur une étude sur les propriétés physiques de la glace de mer et divers autres exposés sans rapport direct avec l'océanographie.

(1) « Coeffeient of eddy viscosity » dans la terminologie anglo-saxonne. On dit parfois en français : « coefficient de turbulence $\gg$. 


\section{b) Symposium sur la circulation océanique et particulièrement la circulation profonde.}

Ce symposium, placé sous la présidence de H. Stommel, comprenait des exposés sur des problèmes théoriques généraux et des études sur la circulation dans des zones géographiques déterminées.

H. Stommel fit d'abord le point des idées admises sur la circulation océanique profonde. Ce que nous savons dérive de trois sources prineipales :

a) La distribution des masses d'eau (caractérisées par une corrélation déterminée entre la température et la salinité);

b) Le calcul du courant "géostrophique 》 coincidant avec la répartition mesurée des densités;

c) Les mesures directes de courants profonds.

a) Un appoint considérable relativement au point de vue de l'étude des masses d'eau est constitué par la carte, établie par Fuglister, de la répartition des températures, salinités et teneur en oxygène dissous à 4.000 mètres de profondeur (fig. 1). Le graphique des corrélations

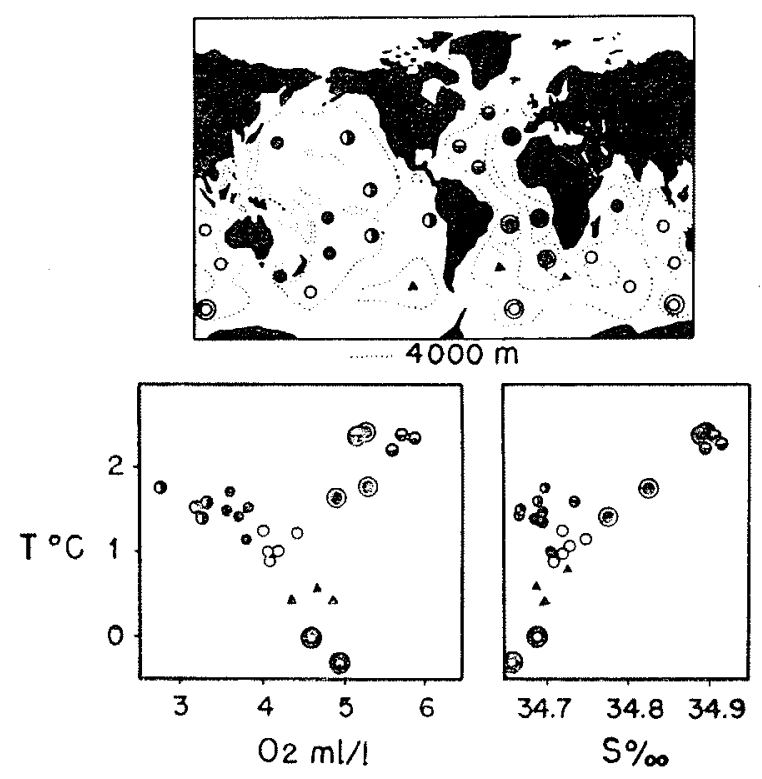

FIG. 1

$\mathrm{S}(\mathrm{T})$ et $\mathrm{O}^{2}(\mathrm{~T})$ permettent de considérer qu'il y a deux « sources » d'eau à 4.000 mètres, eauxtypes ayant des teneurs élevées en oxygène : une eau, assez chaude (plus de $2^{\circ} \mathrm{C}$ ), de salinité élevée $(34,90 \%)$, présente dans le NW de l'Atlantique Nord; et une eau froide $\left(0^{\circ}\right)$, peu salée $(34,70 \%)$, près des côtes de l'Antarctique. Le reste des eaux à 4.000 mètres paraît être un mélange de ces deux eaux-types, la teneur en oxygène décroissant au fur et à mesure que l'on s'éloigne des «sources» dont les eaux envahissent progressivement l'Océan Indien, puis le Pacifique. On a utilisé la décroissance de l'oxygène pour "dater » les eaux marines; mais cette décroissance de l'oxygène n'est pas constante et il vaudrait mieux, pour dater les eaux, disposer de corps radioactifs.

b) Les calculs géostrophiques impliquent le choix - délicat - d'une surface isobare de référence horizontale et sans mouvement, mais Wust a indiqué que ce choix pouvait être guidé par des considérations sur l'hydrologie et a appliqué la méthode avec succès à l'Atlantique Sud.

c) Les mesures directes sont difficiles. Stommel pense que les drogues dérivantes à grande profondeur sont susceptibles de donner des résultats discutables, comme aussi des courantomètres suspendus à des bâtiments mouillés par grand fond. L'apparition des flotteurs de Swallow donne un outil intéressant qui a été déjà employé avec succès. Ils ont récemment mis en évidence, sous le Gulf Stream, un courant inverse portant au SW.

Mais les problèmes de circulation profonde peuvent être abordés théoriquement moyennant diverses hypothèses simplificatrices concernant notamment la permanence du mouvement : on peut tenter d'examiner le régime de circulation profonde provoqué, dans un océan limité par deux méridiens, par une «source»dans chacune des deux zones polaires; dans un système non tournant, on pourrait imaginer un écoule-

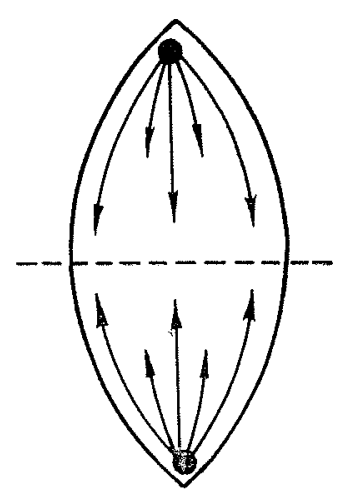

FIG. 2

ment méridien vers l'équateur, pratiquement indépendant de la longitude (fig. 2). En fait, en raison de la rotation, l'écoulement doit être concentré dans une veine collée au bord ouest de l'océan (fig. 3), dans l'un comme dans l'autre hémisphères, avec compensation par des lignes de courant très épanouies en direction N-E à E-N-E (hémisphère nord) ou S-E à E-S-E dans 
l'hémisphère sud; le mouvement de plongée provoquant la «source» est compensé par un lent mouvement vertical d'ensemble vers le haut. Si

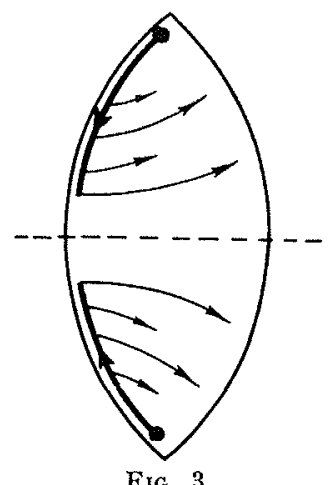

Fig. 3

on se donne seulement une source au nord, la veine profonde entière se prolonge jusqu'au sud, tandis que l'épanouissement-central se produit

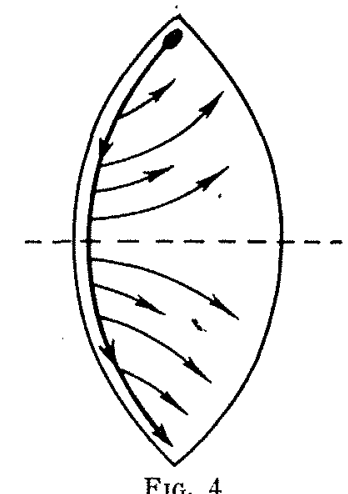

de façon analogue et comprend une composante dirigée ver's le pôle dans chaque hémisphère (fig. 4).
Introduisant les deux sources nord et sud décelées par l'analyse des masses d'eau et des cloisons matérialisant grossièrement les océans,

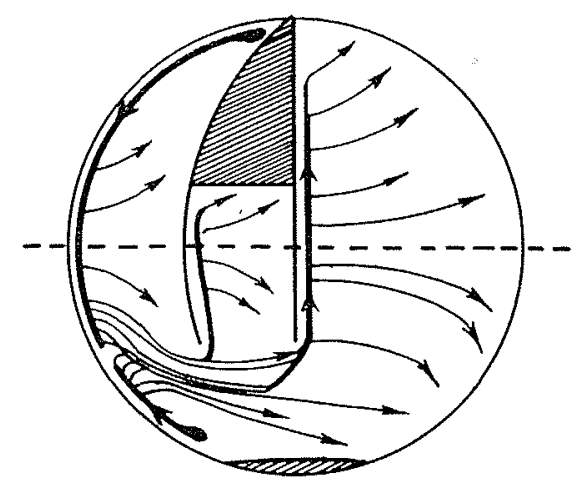

FIG. 5

H. Stommel arrive à trouver un schéma qui doit dévoiler (fig. 5) les grands traits de la circulation profonde. L'eau de la source Nord-Atlantique coule vers le sud le long du bord ouest de cet océan. A la latitude du cap de Bonne-Espérance, cet écoulement rejoint l'écoulement vers le nord provoqué par l'eau plongeant en mer de Weddel; la réunion de ces eaux provoque alors un flux dirigé vers l'est qui envahit l'Océan Indien et le Pacifique en formant une veine de courant portant au nord le long des bords occidentaux de ces océans. Partout, en dehors de ces veines, se rencontre une faible composante vers le pôle.

Les calculs de Wust présentés pour l'Atlantique-Sud confirment ces vues de Stommel; les mesures récentes de Swallow dénotent un courant SW sous le Gulf Stream. On rencontrerait donc une veine de courant nord dans les Océans Indien et Pacifique, qui se distingueraient, par

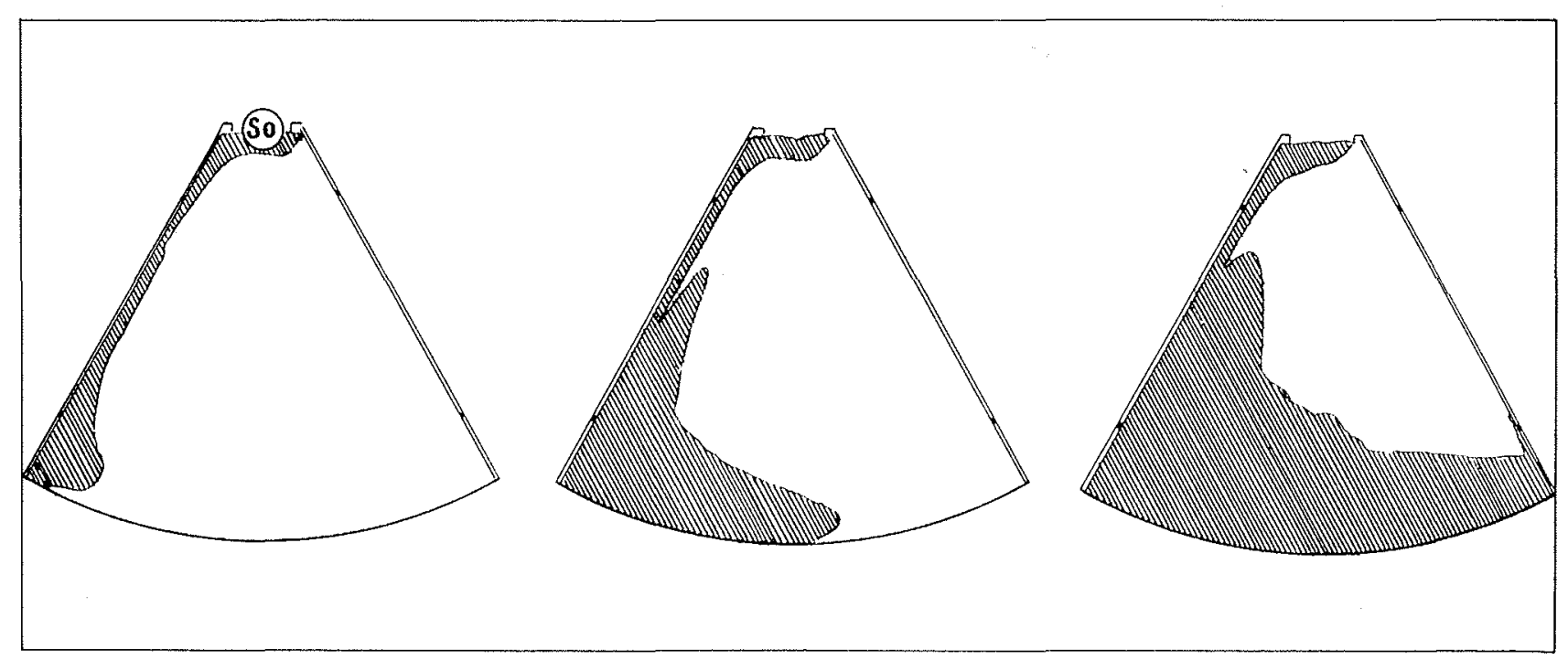

FIG. 6 
ce caractère, de l'Atlantique; en profondeur, dans les parties centrales des océans, il devrait y avoir une faible composante de courants s'ecartant de l'équateur.

Pour vérifier ces idées théoriques de H. Stommel, des études sur modèles réduits tournants ont été exécutées par A. Faller qui travaille à Woods Hole. I' « océan » est un secteur triangulaire de $60^{\circ}$ d'ouverture occupant l'hémisphère nord sur $60^{\circ}$ de «longitude » (fig. 6).

Par analogie avec le schéma d'une «source» d'eau dans la partie arctique, on introduit une source d'eau teintée dans le nord et, puisque l'on ne peut, sur un même bassin tournant, représenter les deux hémisphères (qui devraient tourner en sens inverse), un puits, constitué en fait par une simple élévation du niveau, que l'on peut considérer comme un puits uniformément réparti sur le bassin. On a obtenu une dérive sud nettement marquée sur le bord \& occidental * de l'océan, avec un lent envahissement de l'océan à partir du coin SW. Ceci est qualitativement en accord avec les résultats thériques de Stommel, pour la partie profonde (au-delà de $2.000 \mathrm{~m}$ environ). Stommel prévoit de même une circulation exactement inverse dans la couche supérieure (à moins de $2.000 \mathrm{~m}$ de profondeur), avec une veine oriertée ver's le nord sur le bord ouest.

Si on étend ce schéma aux deux hémisphères, on peut admettre que la circulation d'ensemble, liée à la plongée d'eau dans le nord et la remontée dans le sud (latitude du cap de Bonne-Espérance), se fera essentiellement au fond par une veine limitée appliquée au bord ouest de l'océan et près de la surface par une veine portant vers le nord contre le même bord ouest, les parties centrales et orientales n'étant parcourues que par des circulations lentes (fig. $7 \mathrm{a}$ ).

Donc, la circulation thermodynamique (ou thermohalyne), décelée par l'analyse des masses d'eau, qui suggère les mouvements verticaux cités, doit, en surface, se faire essentiellement par un veine étroite d'eau en direction nord.

Si on superpose alors à ce schéma le schéma de la circulation due aux vents (fig. $7 b$ ) telle qu'elle résulte des travaux plus ou moins parallèles de Munk, Hidaka et Saint-Guily, on obtient un schéma où l'intensification ouest de la circulation due aux vents est aidée ou contrebalancée en partie, selon les cas, par cette circulation thermohaline (fig. $7 c$ ). Stommel voit dans ce fait l'explication de la dissymétrie de l'intensification du courant superficiel dans l'Atlantique Nord et l'Atlantique Sud, phénomène qui attendait encore une explication que ne fournissait pas la seule considération des courants dus aux vents.

Divers exposés d'autres auteurs expliquent comment des études sur la radioactivité natu- relle des eaux sont susceptibles de renseigner sur la circulation océanique, en plus d'études plus classiques comme celles de la teneur en oxygene et en phosphore. Les études de radioaclivité présentées portaient sur le carbone-14, le radium et l'oxygène-18. Il semble d'abord que la teneur de l'eau de mer en ces substances, qui portent en elles leur échelle de temps, sont susceptibles de caractériser les eaux, car on obtient souvent des valeurs différentes dans les différents bassins et dans les différentes « masses d'eau 》 classiquement définies par leur relation $\mathrm{T}-\mathrm{S}$. Mais quand il s'agit d'interpréter ces valeurs pour en déduire le mouvement des eaux, ou plutôt leur vitesse, on se heurte toujours à la dif-

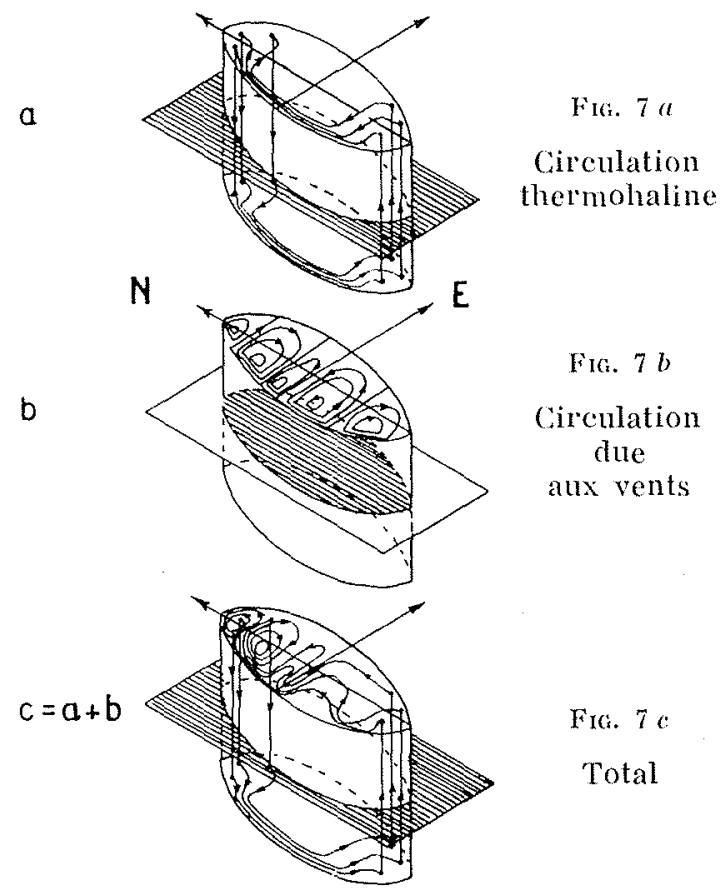

ficulté de savoir si la teneur présente en un point est due à un transport effectif d'eau ou à une simple diffusion du corps radioactif dans le milieu (Broecker, Koczy, Benson). Les méthodes plus classiques de la teneur en oxygène (Worthington, Cooper) et en phosphore (Redfield), bien que se heurtant aux mêmes difficultés, doivent concourir, en liaison avec la méthode usuelle des diagrammes $\mathrm{T}-\mathrm{S}$, à caractériser et à suivre les trajectoires suivies par les eaux (à défaut de la connaissance de leur vitesse sur ces trajectoires); on peut espérer que des éléments plus précis pourront être acquis dans ces analyses des masses d'eau quand le salinometre conductimétrique de Woods Hole sera plus largement employé et, aussi, quand la thermodynamique des mélanges d'eaux diverses et les effets des non-linéarités des équations du mou- 
vement et de l'équation d'état de l'eau auront été plus étudiés (Fofonofí). Il faut noter que la teneur en particules (Jerlov) semble apte à caractériser une eau. Les méthodes théoriques gardent leur intérêt (Saint-Guily) et on peut espérer que de la conjonction des efforts dans les diverses voies évoquées sortiront des vues nouvelles.

Peut-être est-ce faire preuve d'un optimisme raisonnable de dire que les grandes lignes de la circulation océanique permanente (permanence mise en évidence par Fuglister, qui a comparé les résultats de coupes hydrologiques faites par la « Meteor » il y a trente ans et le «Crawford » cette année), sont maintenant connues, essentiellement grâce aux travaux et aux nombreux exposés de H. Stommel, le spécialiste bien connu de Woods Hole. Mais seules les grandes lignes sont inventoriées; de multiples difficultés de détail demeurent et souvent, même dans des eaux très homogènes, on a mesuré des courants appréciables et très fluctuants, notamment près du fond (Mosby). Et même les méthodes géostrophiques classiques ne sont pas à l'abri de discussion (Hidaka), malgré les beaux résultats acquis par Wust pour l'Atlantique Sud.

Des exemples de variations saisonnières remarquables ont été présentés par G. Diétrich, sur le seuil séparant les Faeroe et l'Islande et aussi dans l'étroite vallée sous-marine, profonde de 600 mètres, qui coupe le seuil Islande-Groënland. On constate par moment, sur les seuils, des écoulements vers le sud d'eau arctique froide; cet écoulement cesse par moments, puis peut reprendre; la périodicité de ces fluctuations pourrait ètre de l'ordre de un mois.

Un aspect statistique des diverses valeurs de la température et de la salinité dans les océans a été présenté par Montgomery, Pollak et Cochrane.

\section{c) Symposium sur le transfert de la quantité de mouvement de l'atmosphère à l'eau.}

Ce symposium était présidé par M. H. Charnock, du National Institute of Oceanography de Wormley. L'étude détaillée de récents enregistrements de la répartition verticale des vitesses de vent dans les premiers mètres au-dessus de l'eau a permis à l'auteur une synthèse de ses propres résultats et d'autres obtenus soit en mer, soit en laboratoire. Il en a déduit, pour le cas d'un équilibre de température, une expression mathématique approchée comportant, outre le terme logarithmique usuel de la cote au-dessus de l'eau, un terme sans dimension de la forme $g z / u^{2}$ ( $z=$ cote; $u=$ vitesse du vent), qui permet aussi le calcul de la force d'entraînement superficiel à partir d'une mesure de vent à une seule élévation. Pour des conditions thermiques stables ou instables, l'auteur a proposé des expressions approchées pour évaluer les mêmes grandeurs.

C.H.B. Priestley a présenté les travaux effectués en Australie par E.L. Deacon, qui se relient d'ailleurs à ceux qui ont été évoqués à l'occasion de l'étude de l'évaporation (voir plus haut). Par le relevé du profil de vent près de l'eau, et par l'estimation des tensions de Reynolds grâce à l'analyse des fluctuations horizontales et verticales, cet auteur a obtenu des valeurs cohérentes du coefficient classique $\tau / \rho u_{10}{ }^{2}$ (valeur de 0,0022 pour une vitesse du vent de $10 \mathrm{~m} / \mathrm{s}$ environ) (1).

G. Wust présente une bouée récemment mise au point en Allemagne et qui permet la mesure du gradient thermique entre la cote $+8 \mathrm{~m}$ et la cote $-7,50 \mathrm{~m}$. On obtient aussi, dans l'air, le profit du vent. La bouée est filée d'un bâtiment qui se tient à une cinquantaine de mètres; un câble électrique multiple permet l'enregistrement à bord; l'absence d'effet du navire sur les mesures est ainsi assurée.

$P$. Groen expose ensuite les résultats de travaux qu'il a mené avec P.H. Weeninck sur l'effet du vent et du courant de dérive sur le niveau des mers littorales. L'analyse faite conduit à séparer l'effet propre du vent et les effets de la force de Coriolis et de la force de frottement sur le fond. Par une schématisation raisonnable, le problème se prête assez bien au calcul moyennant détermination empirique de divers facteurs.

Un exposé théorique de Fjeldstad montre qu'au prix d'hypothèses vraisemblables, on peut arriver à montrer que le coefficient de viscosité (vertical) à faire intervenir dans les calculs d'onde internes, varie en raison inverse de la stabilité de l'eau. Stewart, qui examine les effets sur les ondes de gravité des tensions de Reynolds, insiste sur divers effets des échanges d'énergie entre la part turbulente du mouvement et le mouvement moyen.

\section{d) Symposium sur la construction des cartes cotidales.}

Ce symposium n'a pu être présidé, comme prévu, par l'éminent spécialiste qu'est le $\mathrm{D}^{\mathrm{r}} \mathrm{A} . \mathrm{T}$. Doodson, qui a pourtant exposé, dans une note diffusée avant l'Assemblée, diverses idées sur la facon dont on peut, dans des mers peu étendues, calculer la marée au large à partir des données à la côte. Pour le même cas, Rossiter met en garde contre les incertitudes pouvant

\footnotetext{
(1) $\tau=$ force de frottement superficiel,

$\mathrm{o}=$ densité de l'air,

$u_{i 0}=$ vitesse du vent à $10 \mathrm{~m}$ au-dessus de l'eau.
} 
résulter d'une précision insuffisante des observations de marée et sur les effets possibles de diverses configurations du fond. Après quelques exposés très pratiques (Gordon, Farquharson), furent présentés les exposés de MM. Vantroys et Bonnefille, qui ont déjà été discutés à la S.H.F. et qui constituaient la part la plus originale des contributions à ce symposium.

\section{e) Symposium sur les courants de marée au fond dans les eaux côtières et les estuaires.}

Dans un texte diffusé avant l'Assemblée générale, le Président, le $D^{r}$ A.T. Doodson, examine les principaux problèmes qui se posent dans le domaine en question : profil des vitesses, frottement sur le fond, transport de matériaux près du fond. La mise au point de mesureurs instantanés de la vitesse du courant, mécaniques ou électriques, a rendu possible des mesures plus détaillées du champ de courant et de ses fluctuations à courte période d'où d'intéressants apercus sur les difficiles problèmes de la turbulence, du mélange, sur l'effet des couches, etc.

K.F. Bowden expose ensuite les résultats de mesures faites dans l'estuaire de la Mersey et au large d'Anglesey, au noyen d'un courantomètre Doodson et d'un courantomètre électromagnétique (avec champ magnétique extérieur - done analogue à celui présenté déjà à la S.H.F. par M. Remenieras) : le profil de vitesse trouvé est parabolique, sauf tout près du fond ou il devient logarithmique; de sa forme on déduit la force de frottement sur le fond, ainsi que d'intéressantes indications sur la variation du coefficient de turbulence à mesure que l'on s'éloigne du fond. $\mathrm{Si}$ on calcule le coefficient du frottement correspondant à la valeur moyenne de la vitesse entre le fond et la surface, on obtient la valeur du coefficient de frottement $k=0,018$. L'étude des fluctuations turbulentes a permis la détermination des valeurs moyennes du produit - $\bar{u} \bar{u} \bar{w}$ (tension de Reynolds) et a conduit à des valeurs du frottement sur le fond en accord avec les autres estimations faites.

H. Charnock, dans des études parallèles, mesure le courant près du fond au moyen d'un système de 4 courantomètres superposés. Il trouve aussi une loi logarithmique de variation du courant et il en déduit le frottement superficiel correspondant; les valeurs trouvées sont en accord avec celles déduites par Bowden et Fairbairn, d'une méthode dynamique fondée sur des mesures concomitantes de courant et de hauteur d'eau. De nouvelles déterminations par les deux derniers auteurs cités, faites au moyen de l'analyse des fluctuations turbulentes, les ont amenés à des valeurs à peu près doubles. Ce désaccord n'a pas encore été expliqué.
Outre divers exposés strictement «géographiques », c'est-à-dire relatant les résultats de mesures faites notamment dans les détroits de Colombie Britannique, divers auteurs présentèrent une interprélation de ces mesures, notamment Rattray, qui, se fondant sur des mesures de courant à diverses immersions, obtint une évaluation des tensions de Reynolds. J.N. Carruthers présenta ensuite les derniers appareils de mesure de courant qu'il a inventés et qui sont essentiellement concus pour fournir des indications dignes de foi même par mauvais temps, c'est-à-dire au moment où les autres appareils sont généralement déficients et où, pourtant, les mesures sont particulièrement indiquées dans les études de régime des côtes. Dans ce domaine, R.L. Miller présenta ses idées sur les méthodes fondées sur la distribution granulométrique des sédiments pour étudier le courant ou plutôt l'effet global sur le fond de toutes les catégories de courant présentes.

Bowden lut ensuite une note de Sir Claude Inglis et F.H. Allen relative aux courants de fond et au transport de sédiments dans l'estuaire de la Tamise. Des mesures prolongées de courant à diverses profondeurs permirent de relier la vitesse moyenne à la vitesse au fond, à miprofondeur et en surface; on note, près du fond, dans la partie basse de l'estuaire, un transport d'eau vers l'amont, dont la valeur moyenne est d'environ 1 mille par cycle de marée. Mais dans les parties vaseuses de l'estuaire, les transports dans les deux sens sont à peu près équivalents. Le transport de sédiment fut aussi étudié en liaison avec les cycles de marée; les résultats sont différents en vive eau (transport vers l'amont) et en morte eau (transport vers l'aval). Les résultats donnés par des traceurs radioactifs dans la nature et ceux donnés par des grains de celluloïd dans un modèle s'accordaient fort bien. Les transports locaux vers l'amont ont été aussi reproduits par le modèle dans les zones où on les rencontre dans la nature, le mouvement vers l'amont n'étant plus noté dans les régions vaseuses, qui seraient donc celles où ce transport vers l'amont, près du fond, 's'annulerait.

Des études très étendues portant sur les mêmes phénomènes de transport de sédiment ont été faites aux Pays-Bas par Arlman, Santema et Svasek. Leur's méthodes d'attaque du problème sont probablement une synthèse de toutes celles qui existent. Les traceurs radioactifs ont été employés sur une grande échelle dans le cadre d'études généralisées mettant en cuvre des moyens considérables.

Dans le sud de la mer du Nord, les hydrographes allemands ont effectué aussi de fort intéressantes et très denses mesures de courant de longue durée sur des normales à la cote et ont, en particulier, mis en évidence l'influence des 
marées sur la répartition de la salinité au voisinage du littoral.

Cette série de symposiums épuisée, des communications sur des sujets divers étaient prévues. La variété des sujets présentés rend difficile ou très longue une analyse de ces communications, mais on peut cependant tenter de dire un mot des principaux sujets abordés.

Les océanographes canadiens ont exposé les résultats d'importants travaux au large des côtes orientales et occidentales de leur pays (Campbell et Collin, Mc. Lellan, Lauzier et Trites, Pickard).

La question du niveau moyen des mers et de la pente côtière du niveau marin (Descossy, présentée par H. Lacombe) a été l'objet d'une réunion ultérieure des océanographes et des spécialistes du nivellement; les uns comme les autres sont intéressés par la connaissance de ces niveaux, et, faute de liaisons suffisantes entre eux, une lacune demeurera dans le domaine de chacun d'eux. L. Vantroys eut l'occasion, au cours de cette réunion, de présenter aux géodésiens un exposé thériqué sur ces liaisons entre la pente du niveau des mers et les caractéristiques (vitesse et accélération) du mouvement des eaux. Cet exposé illustre l'intérêt de la décision de l'Association Internationale de Géodésie de se livrer à une compensation d'ensemble des divers réseaux européens de nivellement, en sorte que l'on puisse rapidement estimer les pentes côtières de la surface de la mer, ce qui est d'un essentiel intérêt pour les océanographes; et, inversement, ceux-ci peuvent aider les géodésiens dans la difficile question du raccordement des niveaux côtiers de part et d'autre d'un bras de mer. En même temps, il était utile de souligner devant les spécialistes du nivellement le fait que le niveau moyen de la mer en divers lieux est loin de coincider avec une surface de niveau; le raccordement de ces niveaux ne peut se faire - pour la mer - que par une connaissance approfondie des courants marins existant dans la mer étudiée, c'est-à-dire d'un nombre considérable de facteurs.

Un exposé de $\mathbf{T}$. Ichye sur le comportement des tsunamis provoqués par une impulsion du fond de la mer, fournit d'intéressantes indications sur les mouvements auxquels il faut s'attendre et la périodicité des oscillations de niveaux constatées en un point. Les idées de R. Miche (présentées par H. Lacombe) sur le développement asymptotique de l'agitation superficielle de la mer due aux vagues et à la houle peuvent, dans une certaine mesure, etre rapprochés de celles d'Ichye. La méthode préconisée par Miche pour la prévision de la houle à partir de l'agitation existant à la limite du fetch à la cessation de l'action du vent ajoute un nouvel élément relativement simple et constructif aux méthodes actuellement existantes.

Les résultats préliminaires de croisières en Méditerranée et en mer Egée à bord de la «Calypso » et dirigées par H. Lacombe et P. Tchernia sont ensuite présentées : elles mettent en évidence l'extrême complexité du régime de la mer Egée et des régions adjacentes de la Méditerranée, l'importance du cycle annuel (par comparaison avec des ohservations faites vers 1948 par l' «Atlantis ») et donc, des influences climatiques. Les valeurs profondes des températures et salinités en été à $1.000,1.500$ et 2.000 mètres sont présentées sur une carte qui montre nettement l'influence de la mer Egée et permet de suivre l'évolution de ces eaux dans les deux bassins de la Méditerranée.

T. Ichye présente une analyse très fournie de la circulation et de l'hydrologie de la partie NW du Pacifique, fondée sur de nombreuses observations effectuées par les Japonais entre 1932 et la guerre. En particulier, les systèmes de courants subtropicaux déduits des topographies dynamiques de la surface (référence 1.000 décibars) ne seraient pas, comme dans l'Atlantique, strictement zonaux et il existerait un déplacement vers le nord du système entier pendant l'hiver boréal. Les variations séculaires du Kuros. hio ont été étudiées; le régime se rattache à deux schémas principaux : on rencontre soit une zone cyclonique au sud du Japon, soit un écoulement rectiligne en direction de l'est au large du Japon. Le premier schéma, accompagnant une remontée d'eau froide provenant des grandes profondeurs, peut durer plusieurs années, mais l'intensité du tourbillon subit des variations saisonnières considérables.

Diverses régions de l'Océan Indien furent l'objet d'exposés, d'abord de la part de E.C. Lafond, qui a participé à des études effectuées par l'Université d'Andhra sur le plateau continental longeant la côte orientale de l'Inde; le régime des courants, la constitution et le modelé du fond et le régime de la côte dans les différentes saisons ont été particulièrement étudiés. Les travaux de P. Tchernia (présentés par H. Lacombe) sur la dissymétrie des deux parties NW et NE de l'Océan Indien, au regard notamment du comportement de l'Eau Intermédiaire Antarctique, sont en partie le fruit de l'exploitation d'observations non publiées qui ont été communiquées au Laboratoire d'Océanographie Physique du Muséum par des océanographes britanniques. Cet océan se distingue franchement des autres par cette dissymétrie qui motiverait de plus amples études. Dans la partie méridionale de l'O'céan. Indien, les idées de Wexler sur la Con- 
vergence Antarctique ont été l'objet de discussions animées, malheureusement en l'absence de l'auteur.

Dans le domaine des études optiques, on entendit des exposés, très remarqués, de nos deux compatriotes, Mlle J. Lenoble et A. Ivanov, d'une part sur l'absorption du rayonnement ultraviolet dans la mer, et l'étude théorique de la pénétration du rayonnement, et d'autre part sur le degré de polarisation du résidu sous-marin de lumière du jour et la transparence de la mer en divers points des abords des côtes françaises.

Enfin, dans le domaine des analyses chimiques, la détermination faite par J. Lyman des constantes de dissociation des acides carbonique et borique dans la mer est susceptible de faire faire d'importants progrès.

Commentaire de M. le Président BarriLlon :

M. le Président remercie M. Lacombe d'avoir présenté d'une façon très vivante et très précise tout ce qui a été fait à l'Assemblée de Toronto, et se déclare très heureux de voir que des travaux français etaient souvent cités, notamment des travaux qui avaient été présentés à la
Société Hydrotechnique de France.

Il espère, d'autre part, qu'il y aura à la S.H.F. quelques nouvelles communications sur les nombreuses questions présentées à ce Congrès.

\section{NOTRE FRON'TISPICE}

(Cf. page 298)

DE SPARRE (1849-1933).

La carrière du comte Magnus de Sparre est un exemple de la

"polyvalence" caractéristique du tempérament français. Orfier, administrateur, professeur, chercheur, ses travan. matiques pures, balistique, hydrouliques

matiques pares, balistique, hydraulique. p̀̀ court passage A sa sortie de 1Ecole Polytechnique, après un court passage Sciences le Sciences de Lyon, dont il devientra le doyen, et ahi il prepara ì la surface de la terre 》qu'il soutint devant la Faculté des Sa the de paris.

Sa theorie des fonctions elliptiques est restée classique. Ses et h trajectoire des obus i très longue portée. Mais e' surtout en hydraulique, dans le domaine des surpressions dans les con en hydratiliqu, dans le domaine des surpressions dans les conduites et des couns de bélier qu'il s'est imposé à l'attention des Ingenteurs. Coordonnant les theses de Rateau et d'Allievi, ll cations à l'A Académie des Sciences et à la Société Hydrotechnique de France. M. Ch. Camichel a pu dire, de son mémoire que de France. M. Ch. Camichel a pu dire, de son mémoire modèle de clarté, de précision et d'élégance $\gg$. La condition dite « de Sparre » a précisé la notion aujourd'hui classique de «fermeture lente».

\section{DE SPARRE (1849-1933).}

Comt Magnus de Sparre's career is an example of the poly. valent French temperament.

He was an officer, an administrator, a teacher and a scientist and his scientific work covered widely different fields such a pure mathematics, ballistics and hydranlics.

He spent a short time in the Artillery after leaving the Ecole Polytechnique and then began professor at the Catholic Facult of Sciences at Lyons University of which he later became the Dean. At Lyons he prepared his zoll known thesis "on the motion of a conical pendulum at the carth's surface" and ho presented it at the Paris Faculty of Science.

His theory of elliptic functions has become classic. In ballistics he dealt with the motion of projectiles and arith the trajectories of shells from very long range guns $B$ ut he is best hnown of shells from very long ange gunts. But he is best knowon as hydrathic engineer, in which capacity he did research in con nection with pressure surges in conduits and water hammer. He coordinated the work of Ratean and Allievi and developed their

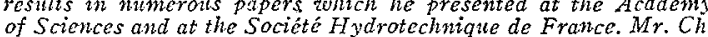
Camichel said of his paper on multiple characteristics conduits, that it was an clarity, accuracy and elegance. What is known as the "de Sparre" condition, clarified the now classic idea of "slow closure". 